\title{
A Study on CS-RZ-ASK Modulation Format for Long Haul Optical Communication
}

\author{
Parneet Kaur ${ }^{1}$, Dr. Hemant Purohit ${ }^{2}$, Guneet Singh ${ }^{3}$ \\ M.E. Student \& Research Scholar, Department of ECE, MBM Engineering College, Jodhpur, Rajasthan, India ${ }^{1}$ \\ Associate Professor \& Head, ECE, Jodhpur Institute of Engineering and Technology (JIET), Jodhpur, Rajasthan, India ${ }^{2}$ \\ Student, Department of EEE, SRM University (Ramapuram Campus), Chennai, India ${ }^{3}$

\begin{abstract}
This paper evaluates the performance of CS-RZ-ASK modulation format in optical communication by presenting the Q-factor, Bit Error Rate and noise margin at different distances. This technique suppress the power of carrier that carries no information in order to increase the efficiency in terms of power saving.
\end{abstract}

Keywords: Bit Error Rate; Eye Diagram; Mach Zehnder Interferometer Modulator; Quality Factor.

\section{INTRODUCTION}

The direct transmission of the binary data without any transformation in frequency is known as Baseband Modulation or line codes. A variety of pulse waveforms are utilised for representing the digital sequences. There are two major types of line codes are RZ and NRZ. Return to Zero (RZ), in this type of line code the waveform pattern returns to zero level in one bit interval while in Not Return to Zero (NRZ), the waveform pattern does not return to zero level for the complete bit interval. Depending upon the voltage level assigned, the line codes are further divided into Uni polar signalling, Polar signalling, Manchester signalling and Bipolar signalling. Advantages of line codes over the modulation format are adequate timing information, spectrum that is suitable to the channel, narrow bandwidth, low error probability, error detection capability, transparency and differential coding [4]. Modulation is a process used for efficient transmission of information over a medium. When the information is converted to send it successfully through an optical fiber is known as optical modulation. There are three basic techniques: ASK, PSK and FSK. In Amplitude shift keying (ASK), the amplitude of the light carrier wave generated by an optical source is changed according to the digital data. Suppose for transmitting ' 1 ', the light wave carrier is transmitted at particular amplitude and for ' 0 ' the amplitude is changed. Depending upon the occupation of the state ' 1 ', optical RZ and NRZ can be produced. For $\mathrm{RZ}$, the digital data is transmitted about half the bit interval while for NRZ the digital data is transmitted over entire bit interval. In Phase shift keying, the light wave carrier is changed in phase for transmission of digital data. The phase here indicates the shift in the angle at which the sinusoidal carrier starts. For ' 0 ' the phase would be shifted by ' $180^{\circ}$ ' and for transmitting ' 1 ', there is no change in phase. In Frequency shift keying, the frequency of the carrier represents the digital information. One particular frequency is selected for the transmission of ' 0 ' and another for ' 1 '. FSK can be considered as continuous phase continuous phase modulation.

Hybrid transmission techniques like QAM can also be used for transmission by combining the basic modulation

technique of ASK and PSK that is both the amplitude and phase of light wave carrier changes for transmission of digital data [2].

External optical modulation techniques can also be used for transmitting to preserve the line width narrowness so that only the sidebands dominate in the spectrum of the pass band characteristics. By applying a pulse pattern generator and laser output to the electro optic modulator using the principle of interferometric constructive and destructive interference to represent the on-off states of the diagram, NRZ optical pulses can be produced. When this NRZ pulses is again fed into the external modulator, the output results into RZ optical pulses. This second modulator is known as pulse carver. In Rx optical line code, for ' 1 ' the light wave is present for the portion of bit interval and for ' 0 ' no light is present in entire bit interval. In optical communication, the suppression of carrier give rise to the CS-RZ modulation. It is achieved by biasing the modulator in such a way that the carriers passing through the two parallel paths of an interferometric modulator are $180^{\circ}$ phase shift difference with each other so that carrier is suppressed at the centre frequency. This suppression can be achieved by adjusting the biasing points at the minimum and maximum transmission point of the transmission characteristics of Mach Zehnder Interferometer Modulator. There are two types of RZ pulses depending upon the driving voltage and the basing schemes.

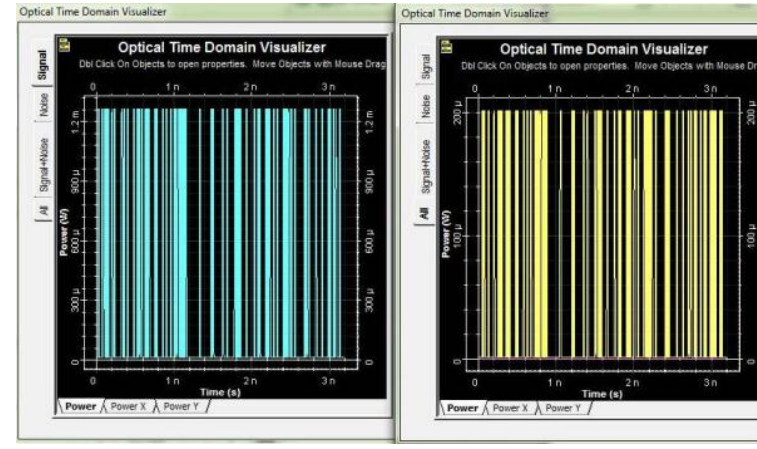

Fig 1: RZ and CS RZ Optical Pulses. 
The $33 \%$ duty cycle RZ pulses is known as RZ33 pulse whereas $67 \%$ duty cycle RZ pulses is known as CS RZ pulses. The bias points control the DC voltage so that operating points stay at the same quadrature point. DC drift occurs due to the build up of charges on the surface of the crystal substrate. This causes the operating point to shift from the quadrature point or from minimum or maximum locations on the transfer curve [2].
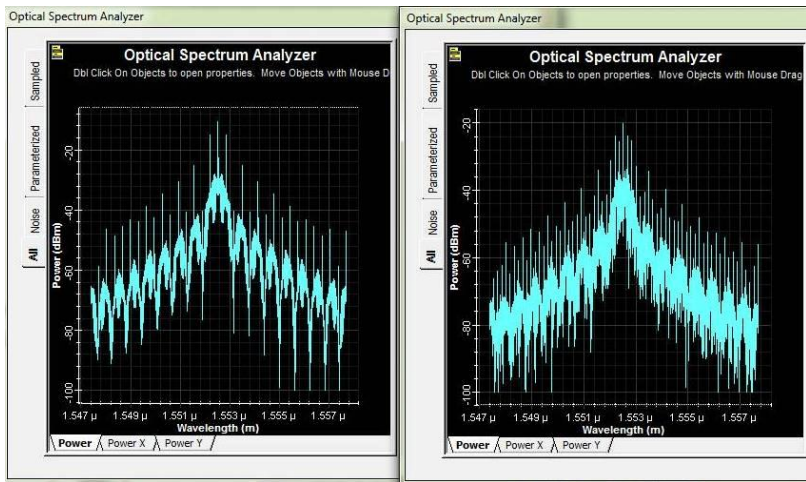

Fig 2: RZ and CS RZ Optical spectrum.

\section{CIRCUIT DIAGRAM}

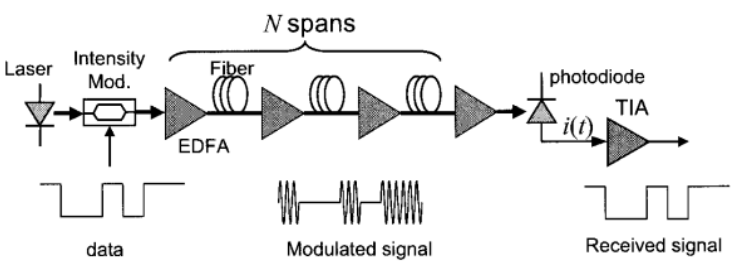

Fig 3: Basic Optical Communication System [5]

The above figure shows the basic components used in the optical communication system. In this system, the digital data is carried in the light intensity called on-off keying or intensity modulated system. The optical source like laser is used for light wave carrier whose amplitude, phase or frequency is changed using an external modulator for the transmission of bits. After the modulation is signal passed through the amplifier to boost up the power and then launched to optical fiber. This optical signal is converted into the electrical signal using a photodiode. It basically converts a photon to an electron. The transimpedance amplifier is used to convert photocurrent to voltage [5]. CS RZ transmitter and receiver is implemented on the basis of this optical communication system. In transmitter side, a laser of $193.1 \mathrm{THz}$ and $4 \mathrm{~mW}$ power is used for carrier wave and the RZ electrical pulse generator is applied to the external modulator to get RZ optical pulses. The output of first modulator is applied to second modulator to get suppressed carrier. The modulator used is LiNbO3 Mach Zehnder Modulator. It works on the principle of Mach Zehnder Interferometer where the light beam is spitted into two paths. If any one of the path is phase shifted then the destructive interference takes place at the combiner otherwise constructive interference is achieved. In $\mathrm{LiNbO}_{3}$ optical MZIM, $3 \mathrm{~dB}$ Y splitter and combiner are used where phase shift is induced in one of the path by changing the refractive index through change in the electric field [3].

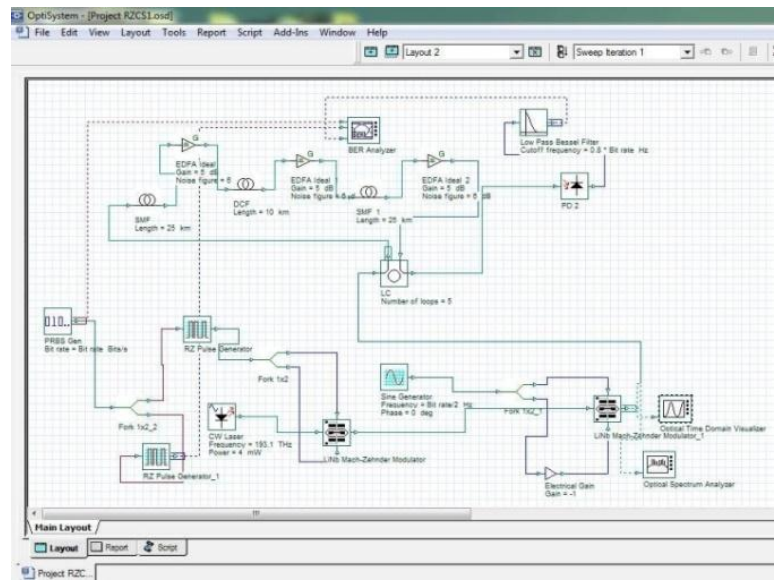

Fig 4: CS RZ Transmitter and Receiver

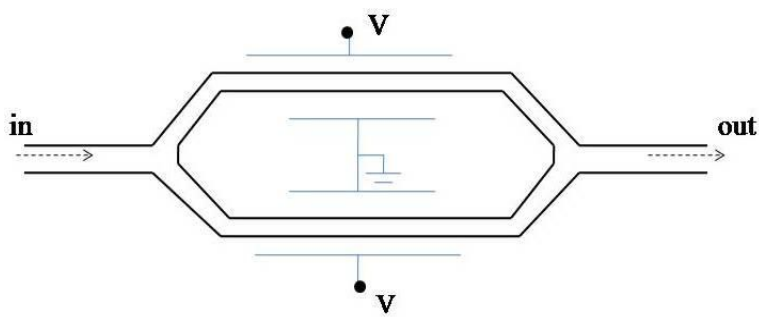

Fig 5: Mach Zehnder Interferometer Modulator

The benefits of using an external modulator in optical fiber communication links are: higher optical output power coupled into the optical fiber, very small pulse broadening, higher transmission rates, longer link distance, fewer EDFA's required, no jitter, electronic noise rejection, $>20$ $\mathrm{dB}$ dynamic range, immune to environmental changes, supports wideband transmission, cost efficient. The $\mathrm{LiNbO}_{3}$ optical MZIM along with EDFA are ideal for applications like synchronous transfer mode (ATM), SONET/SDH optical add/drop multiplexers, undersea systems, CATV, terrestrial communication links $<100$ $\mathrm{km}$, testing and measuring systems, RF down links from satellites, long distance communication system, soliton systems. Optical amplifiers amplify the photons and then transmit them to the next point of amplification through the optical fiber [3].

It differs from the electronic amplifier as they do not require the conversion of photons to electronic signals for amplifying. By deploying the optical amplifier, the distance between optical repeaters increases and the volume of transmitted data does not degrade the amplification process. One of the optical amplifiers used in the communication process is Erbium Doped Fiber Amplifier. EDFA is composed of small amount of optical fiber doped with rare earth erbium $\left(\right.$ Erb3 $\left.^{+}\right)$that has several energy levels. A laser is used to excite the Erbium ions that are initially at ground level. The BER analysers is connected to calculate the parameters like Q-factor, Noise Margin through eye height and BER. The looping system is used to increase the distance of transmission [3].

\section{PARAMETERS}

The CS RZ is studies on the basis of three parameters: Qfactor, BER and Noise margin. 
Quality factor and Bit Error Rate: In the binary digital communication system, there are two signal levels. Each level will have discrete signal to noise ratio due to the difference in average noise associated with them. In order to calculate the overall probability of bit error, both the signal to noise ratio must be considered. The combination of two discrete SNRs into a single quantity providing a convenient measure of overall system quality is called the quality factor [1]. If we compare the sampled voltage $v(t)$ to a reference value $\gamma$ called the decision threshold. If the $\mathrm{v}(\mathrm{t})$ is greater than the $\gamma$, binary one has to be transmitted and if it less than $\gamma$ then binary zero has to transmitted. The major error caused in making the right decision is due to the noise added to the received data assuming perfect synchronisation between bit stream and the bit clock. The probability density function $\mathrm{v}(\mathrm{t})$ with Gaussian noise (AWGN) is given as :

$\operatorname{PROB}\left[\mathrm{v}(\mathrm{t}), \sigma_{\mathrm{x}}\right]=1 \cdot \mathrm{e}^{-0.5(\mathrm{v}(\mathrm{t})-\mathrm{Vs} / \sigma \mathrm{x}) 2} / \sqrt{ } 2 \Pi \sigma_{\mathrm{x}}{ }^{2} \quad$ equation I

Where $\mathrm{v}_{\mathrm{s}}$ is the voltage transmitted depending upon the decision or mean value of the density function, $\sigma$ is the standard deviation of the noise and $\mathrm{v}(\mathrm{t})$ is the sampled voltage in the receiver at time $\mathrm{t}$.

Assuming $\mathrm{v}_{\mathrm{s}}$ can take on only two voltage level than the probability of error in the receiver will be:

$$
\mathrm{P}[\mathrm{e}]=\mathrm{P}\left[\mathrm{v}(\mathrm{t})>\gamma \mid \mathrm{v}_{\mathrm{s}}=\mathrm{v}_{\mathrm{L}}\right] \mathrm{P}\left[\mathrm{v}_{\mathrm{s}}=\mathrm{v}_{\mathrm{L}}\right]+\mathrm{P}\left[\mathrm{v}(\mathrm{t})>\gamma \mid \mathrm{v}_{\mathrm{s}}=\mathrm{v}_{\mathrm{H}}\right] \mathrm{P}\left[\mathrm{v}_{\mathrm{s}}=\mathrm{v}_{\mathrm{H}}\right]
$$

Where $\mathrm{P}[\mathrm{e}]$ is the probability of error. It is assumed that probability of transmitting $\mathrm{v}_{\mathrm{H}}$ and $\mathrm{v}_{\mathrm{L}}$ is equal that is $\mathrm{P}\left[\mathrm{v}_{\mathrm{H}}\right]$ $=\mathrm{P}\left[\mathrm{v}_{\mathrm{L}}\right]=0.5$ then the probability of error becomes:

$\mathrm{P}[\mathrm{e}]=\mathrm{P}\left[\mathrm{v}(\mathrm{t})>\gamma \mid \mathrm{v}_{\mathrm{s}}=\mathrm{v}_{\mathrm{L}}\right] 0.5+\mathrm{P}\left[\mathrm{v}(\mathrm{t})>\gamma \mid \mathrm{v}_{\mathrm{s}}=\mathrm{v}_{\mathrm{H}}\right] 0.5$

$=\frac{1}{2} \int_{\infty}^{\gamma} \operatorname{PROB}\left[\mathrm{v}(\mathrm{t}), \sigma_{\mathrm{L}}\right] \mathrm{dt}+\frac{1}{2} \int_{\gamma}^{\infty} \operatorname{PROB}\left[\mathrm{v}(\mathrm{t}), \begin{array}{c}\text { Equation III } \\ \left.\sigma_{\mathrm{H}}\right] \mathrm{dt} \\ \text { equation IV }\end{array}\right.$

From above equations, the probability of error is equal to the area under the tails of the density functions that extend beyond the threshold $\gamma$. This area is known as Bit Error Rate and it depends upon two main factors: the standard deviations of the noise $\left(\sigma_{\mathrm{L}}\right.$ and $\left.\sigma_{\mathrm{H}}\right)$ and the voltage difference between $\mathrm{v}_{\mathrm{L} \text { and }} \mathrm{v}_{\mathrm{H}}$. For a special case when $\mathrm{v}_{\mathrm{L}}=$ $\mathrm{v}_{\mathrm{H}}$ the threshold is halfway between the low and high levels $\gamma=\left(v_{H}-v_{L} / 2\right)$ and for all other cases the optimum threshold for minimum BER is either lower or higher than $\left(\mathrm{v}_{\mathrm{H}}-\mathrm{v}_{\mathrm{L}} / 2\right)[1]$

Eye Diagram: The effect of channel filtering and channel noise can be seen by observing the received line code on an analogue oscilloscope. We connect the receiver's response to the random pulse sequence on the vertical plates of an oscilloscope, and sawtooth wave at signalling frequency on the horizontal plates. The horizontal base of an oscilloscope is made equal to the pulse duration.

This setup causes the synchronized superimposition of successive symbol intervals into a family of traces in a signal interval $(0, \mathrm{~T})$, giving a eye pattern. If the filtering is absent or the bandwidth corresponding to the transmission of the data pulses is infinite, then a rectangular pulse shape response is observed. For no bit error, the eye will be open otherwise eye will be

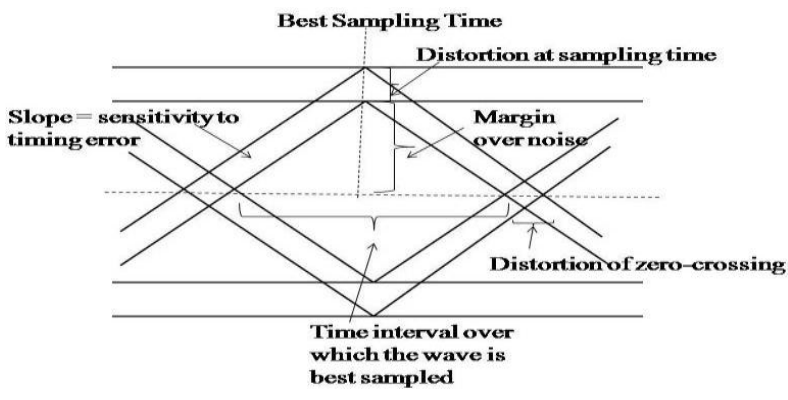

Fig 6: Eye Diagram

closed indicating bit error in the receiver output due to Inter Symbol Interference or noise. It provides an excellent mean to examine the quality of received line code $[1,6]$. It provides following information:

1. The timing error allowed on the sampler at the receiver is given by the width inside the eye called eye opening. The preferred time of sampling is at the point where the vertical opening of the eye is largest.

2. The sensitivity to timing error is given by the slope of the open eye.

3. The noise margin is given by the height of the eye opening.

\section{IV.RESULTS}

TABLE I

\begin{tabular}{|c|c|c|c|c|}
\hline $\begin{array}{c}\text { Number } \\
\text { of loops }\end{array}$ & $\begin{array}{c}\text { Distance } \\
\text { covered }\end{array}$ & $\begin{array}{c}\text { Quality } \\
\text { factor }\end{array}$ & $\begin{array}{c}\text { Minimu } \\
\text { m BER }\end{array}$ & $\begin{array}{c}\text { Eye } \\
\text { Height }\end{array}$ \\
\hline 1 & 60 & $\begin{array}{c}20.466 \\
5\end{array}$ & Very low & $\begin{array}{c}0.00014 \\
580\end{array}$ \\
\hline 2 & 120 & $\begin{array}{c}13.784 \\
1\end{array}$ & Very low & $\begin{array}{c}0.00013 \\
264\end{array}$ \\
\hline 3 & 180 & $\begin{array}{c}10.697 \\
5\end{array}$ & Very low & $\begin{array}{c}0.00012 \\
171\end{array}$ \\
\hline 4 & 240 & 9.2156 & Very low & $\begin{array}{c}0.00011 \\
391\end{array}$ \\
\hline 5 & 300 & 7.9836 & Very low & $\begin{array}{c}0.00010 \\
478\end{array}$ \\
\hline 6 & 360 & 7.1168 & $\begin{array}{c}6.75182 \mathrm{e} \\
-0.13\end{array}$ & $\begin{array}{c}9.643 \mathrm{e}- \\
005\end{array}$ \\
\hline 7 & 420 & 6.3105 & $\begin{array}{c}\text { Very low } \\
0.715 \mathrm{e}- \\
005\end{array}$ \\
\hline 8 & 480 & 5.7343 & $\begin{array}{c}4.19956 \mathrm{e} \\
-010\end{array}$ & $\begin{array}{c}7.890 \mathrm{e}- \\
005\end{array}$ \\
\hline 9 & 540 & 5.3756 & $\begin{array}{c}2.99425 \mathrm{e} \\
-010\end{array}$ & $\begin{array}{c}7.294 \mathrm{e}- \\
005\end{array}$ \\
\hline 10 & 600 & 4.9288 & $\begin{array}{c}1.54705 \mathrm{e} \\
-008\end{array}$ & $\begin{array}{c}6.423 \mathrm{e}- \\
005\end{array}$ \\
\hline 11 & 660 & 4.5795 & $\begin{array}{c}8.02121 \mathrm{e} \\
-007\end{array}$ & $\begin{array}{c}5.628 \mathrm{e}- \\
005\end{array}$ \\
\hline 12 & 720 & 4.3133 & $\begin{array}{c}2.61511 \mathrm{e} \\
-006\end{array}$ & $\begin{array}{c}4.964 \mathrm{e}- \\
005\end{array}$ \\
\hline 13 & 780 & 3.9726 & $\begin{array}{c}2.16675 \mathrm{e} \\
-005\end{array}$ & $\begin{array}{c}3.992 \mathrm{e}- \\
005\end{array}$ \\
\hline
\end{tabular}

\section{CONCLUSION}

The above results show the performance of the RZ-CS modulator in long haul optical communication at various distances. With the increase in distance the Eye Height (Noise Margin) and Quality factor decreases and Minimum Bit Error Rate increases. 
INTERNATIONAL JOURNAL OF INNOVATIVE RESEARCH IN ELECTRICAL, ELECTRONICS, INSTRUMENTATION AND CONTROL ENGINEERING

Vol. 3, Issue 10, October 2015

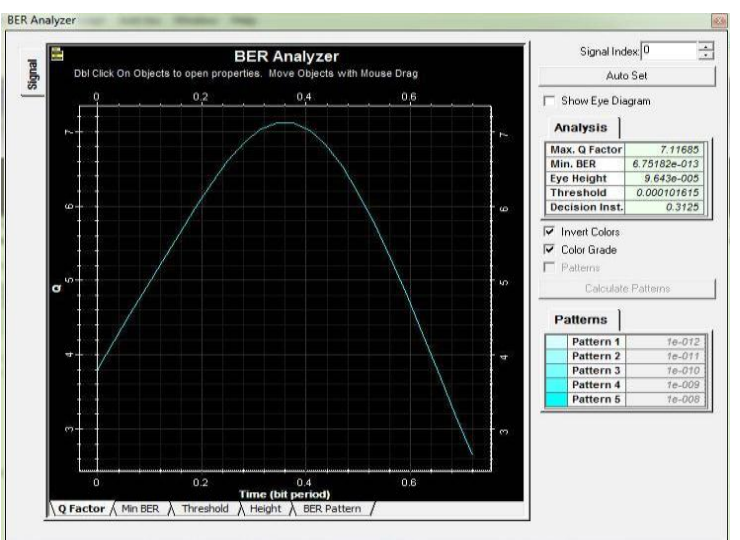

Fig 7: Bit Error Rate Analyser indicating Quality factor $N=6$

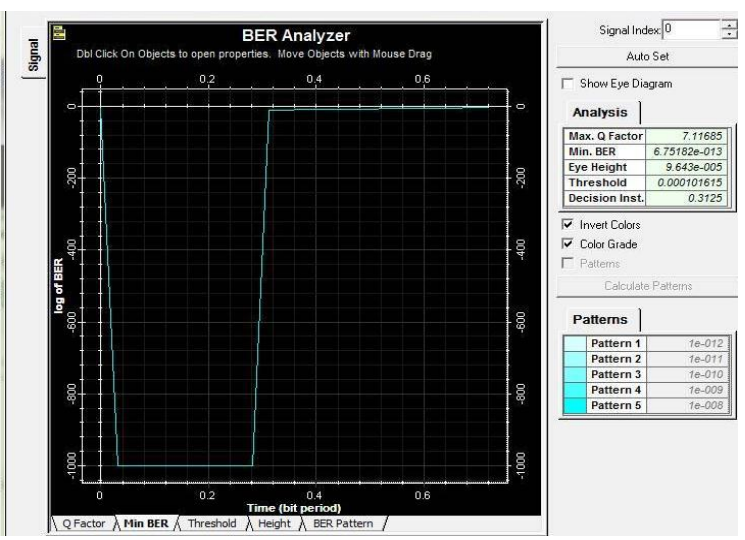

Fig 8: Bit Error Rate Analyser indicating Minimum BER N=6

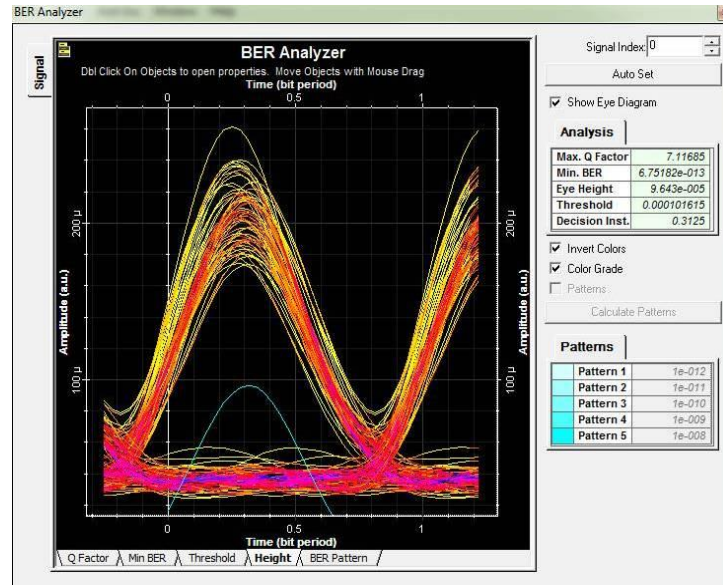

Fig 9: Bit Error Rate Analyser indicating Eye Diagram along with Eye Height for $\mathrm{N}=6$

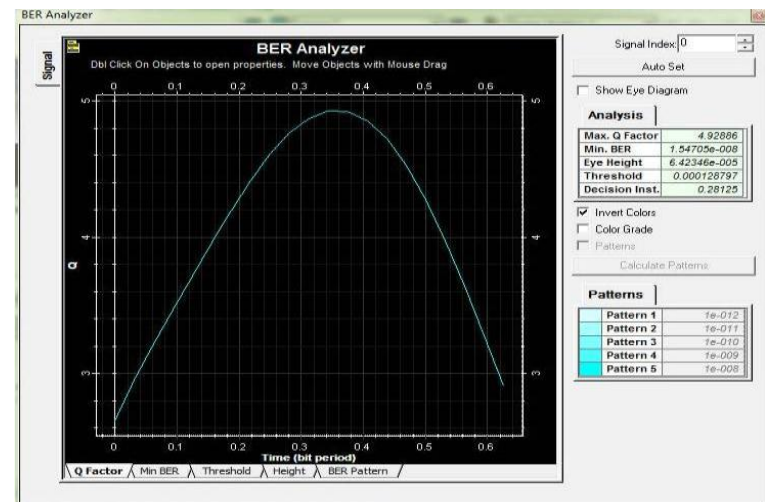

Fig 10: Bit Error Rate Analyser indicating Quality factorN=10

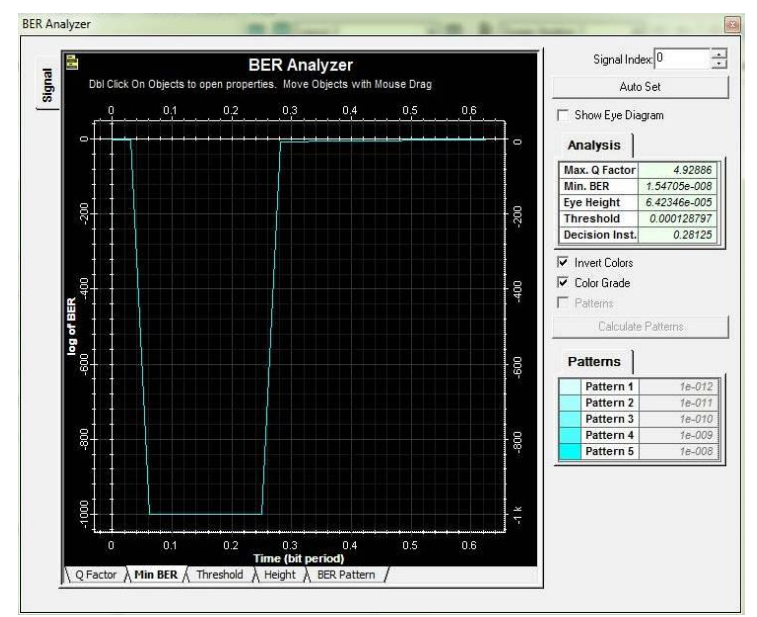

Fig 11: Bit Error Rate Analyser indicating Minimum BER for $\mathrm{N}=10$

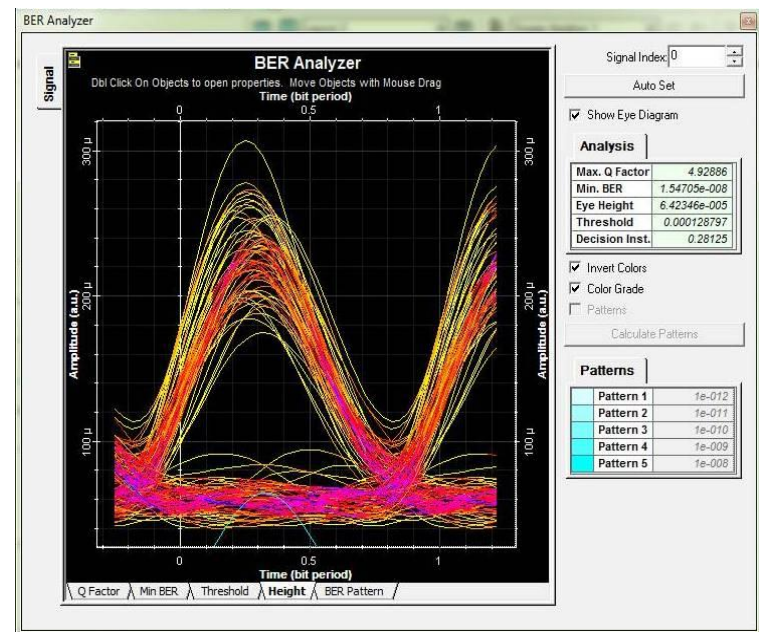

Fig 12: Bit Error Rate Analyser indicating Eye Diagram along with Eye Height for $\mathrm{N}=10$

\section{REFERENCES}

[1] Purohit Hemant, "Study of Coherent Optical OFDM and Performance Evaluation of NRZ-DPSK for Long Haul Optical Transmission Systems", Dissertation work for the degree of Masters of Engineering in Digital Communication Engineering, Jai Narain Vyas University, Jodhpur, 2011

[2]. Le Nguyen Binh, "Digital Optical Communications", CRC Press, Taylor and Francis Group, 2009 ISDN 13: 978-1-4200-8205-0.

[3]. Harold Kolimbiris, "Fiber Optics Communications", Pearson Education Inc. ISBN 978-81-317-1588-8.

[4]. Fuqin Xiong, "Digital Communication Techniques", Artech House 2000 ISBN 0-89006-970-0

[5]. Keang Po Ho, "Phase Modulated Optical Communication Systems", Springer 2005 ISBN 0-387-24392-5.

[6]. Leon W. Couch II, "Digital and Analog Communication systems", Fifth edition, Prentice Hall International, Inc. ISBN 0-13-599028-9. 